\title{
Role of neurosurgeon in the management of COVID 19 patients
}

Recently a significant number of COVID 19 patients had been detected with intracranial hemorrhage in Dhaka. These cases were detected too late. The neurosurgeon's help was sought when there was nothing left for him to do. COVID 19 patients are generally admitted under a pulmonologist and along with an ICU consultant at times. They both are so busy with the management of the lung condition that the intracranial problem skips from their mind. For example a fifty years old male with COVID developed respiratory failure with a low oxygen saturation in blood. He was put on ventilator in the ICU. After two days he was having severe restlessness. His sedation was increased to combat the restlessness. Two days later he was found to have no response when tried for weaning from the ventilator. Then a CT SCAN brain was done which revealed a posterior fossa haemorrhage with gross hydrocephalus.

The neurosurgeon was then called who confirmed brain death. Our Late Prof. Rashiduddin Ahmad of Neurosurgery in his lectures used to say that when an unconscious person is restless think that either the brain is tight or the bladder is tight. This was taught by his teacher Late Prof. Gillingham. Retrospectively, had a proper neurological evaluation been done at the onset of restlessness a neurosurgical intervention could have made a different picture. So lastly I would like to conclude with humble remark that in ICU the management of COVID 19 patients be done with a team of specialists including a Neurosurgeon so that a better outcome is achieved. 